\title{
Award for outstanding contributions to the Chinese Journal of Cancer
}

\author{
Ji Ruan*
}

\begin{abstract}
At the 4th Guangzhou International Symposium on Oncology, Rui-Hua Xu, Chao-Nan Qian, and Wei Zhang-the chairmen and editors of the Chinese Journal of Cancer-announced and presented awards to 14 authors in recognition of their outstanding contributions to the journal.
\end{abstract}

Close collaborations between scientists and physicians from different backgrounds significantly improves our understanding of cancer biology. An excellent example of these collaborations was the 4th Guangzhou International Symposium on Oncology, which was held November 5-7, 2015, in Guangzhou, China. The Guangdong Anti-Cancer Association, the United States Chinese Anti-Cancer Association (USCACA), Sun Yat-sen University Cancer Center, and the Chinese Journal of Cancer (CJC) jointly organized this symposium. More than 1500 cancer researchers and clinicians attended this productive meeting, which included speakers from China, the United States, Italy, France, England, and Japan. The presentations covered several main types of cancer and reported progress in basic, translational, and clinical research.

The aforementioned organizations have jointly organized this symposium on oncology three times now. The first was the 2nd Guangzhou International Symposium on Oncology, held May 20-22, 2011, in Guangzhou, China [1]; the second was the 3rd Guangzhou International Symposium on Oncology, held November 20-22, 2013, also in Guangzhou, China [2].

At this the 4th Guangzhou International Symposium on Oncology, Rui-Hua Xu, Chao-Nan Qian, and Wei Zhangthe chairmen and editors of the CJC-announced and presented awards to 14 authors in recognition of their outstanding contributions to the journal in the past several years.

The CJC Editorial Board members selected the following 14 award winners.

*Correspondence: ruanji@sysucc.org.cn

Chinese Journal of Cancer, Sun Yat-sen University Cancer Center, Guangzhou 510060, Guangdong, P. R. China
1. Webster Cavenee, PhD Ludwig Institute for Cancer Research, University of California-San Diego, USA

2. Khurum Khan, MD, PhD Royal Marsden Hospital, England

3. Zhe-Sheng Chen, MD, PhD St. John's University, USA

4. Takeo Nakanishi, MD, PhD Kanazawa University, Japan

5. Joseph Tien Seng Wee, MD, PhD National Cancer Centre, Singapore

6. Binhua P. Zhou, MD, PhD University of Kentucky School of Medicine, USA

7. Zhong-Zhen Guan, MD, PhD Sun Yat-sen University Cancer Center, P. R. China

8. Wan-Qing Chen, MD, PhD National Office for Cancer Prevention and Control/ National Central Cancer Registry, P. R. China

9. Wen-Qi Jiang, MD, PhD Sun Yat-sen University Cancer Center, P. R. China

10. Wen-Lin Huang, MD, PhD Sun Yat-sen University Cancer Center, P. R. China

11. Zhi-Wei Zhou, MD, PhD Sun Yat-sen University Cancer Center, P. R. China

12. Su-Mei Cao, MD, PhD Sun Yat-sen University Cancer Center, P. R. China

13. Juan Wei, MD, PhD Department of Oncology, Second Affiliated Hospital of Southeast University, P. R. China

14. Yi Wan, MD, PhD

Suzhou Kowloon Hospital, P. R. China

\section{Biomed Central}

(C) 2015 Ruan. This article is distributed under the terms of the Creative Commons Attribution 4.0 International License (http:// creativecommons.org/licenses/by/4.0/, which permits unrestricted use, distribution, and reproduction in any medium, provided you give appropriate credit to the original author(s) and the source, provide a link to the Creative Commons license, and indicate if changes were made. The Creative Commons Public Domain Dedication waiver (http://creativecommons.org/publicdomain/ zero/1.0/) applies to the data made available in this article, unless otherwise stated. 


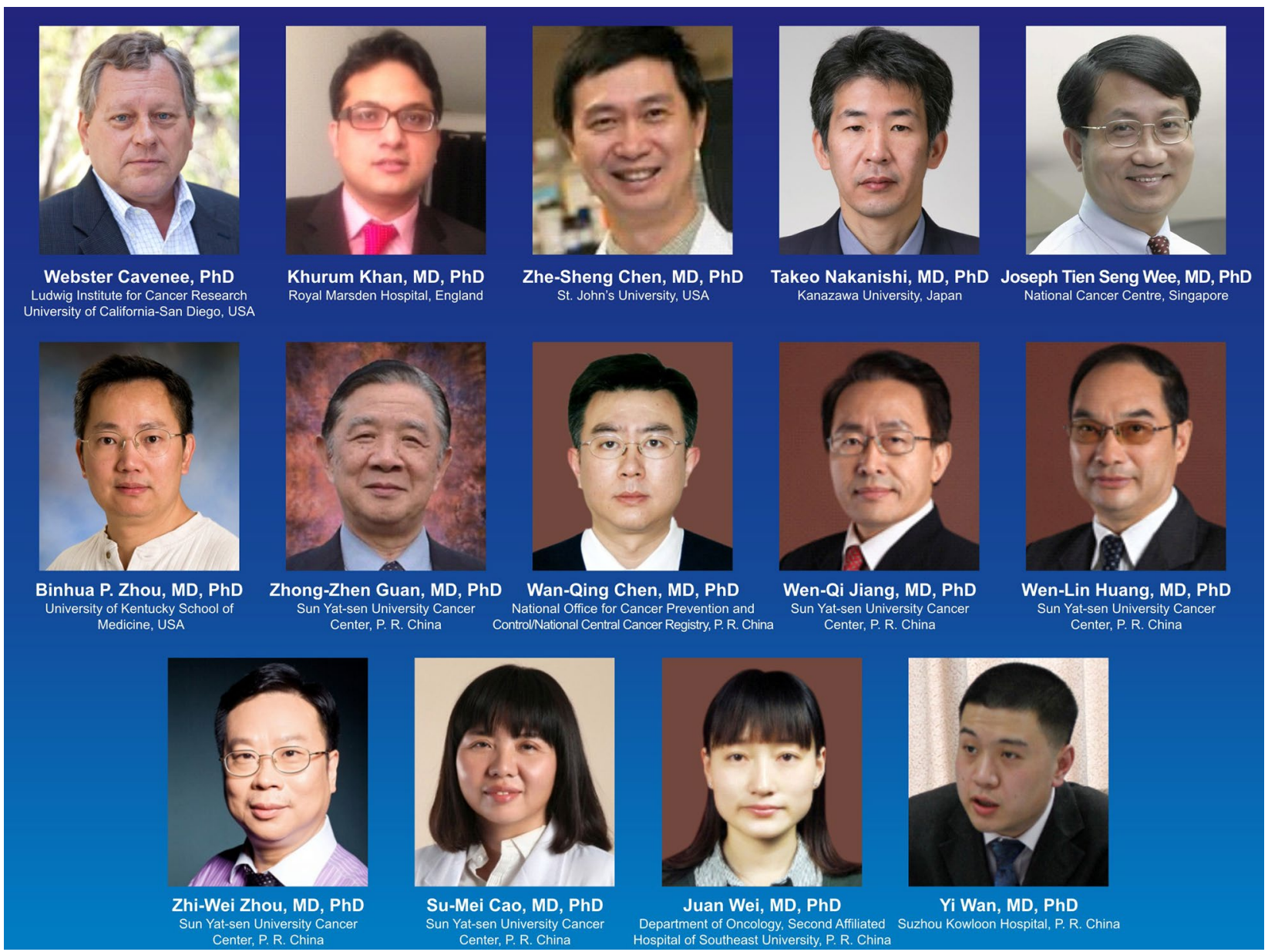

The guidance of the CJC Editorial Board members, the great contributions of reviewers, authors, and readers, and the tireless work of the editorial staff have resulted in papers published in the $C J C$ being cited in many reputable journals. This has markedly increased the journal's international impact. A testament to this came on July 22, 2014, when the CJC editorial office was notified by Thomson Reuters that CJC publications, beginning in 2012, had been accepted for coverage in the Science Citation Index Expanded, which is available at the Web of Science Core Collection, Biological Abstracts, and BIOSIS Previews [3]. The CJCs first impact factor, obtained in June 2015, was 2.155.

The CJC will continue to highlight novel discoveries and technologies in all cancer research fields and relentlessly pursue the ultimate goal of eradicating this disease.

\section{References}

1. Qian $\mathrm{CN}$, Zhang W. Outsmarting cancer: an international brainstorm in Guangzhou. Chin J Cancer. 2011;30:505-7.

2. Xiao WW, Qian CN. Our DREAM of defeating cancer: a summary of the 3rd Guangzhou International Symposium on Oncology. Chin J Cancer. 2014;33:125-32.

3. Zeng YX, Zhang W, Qian CN, Xu RH, Ruan J. The Chinese Journal of Cancer is indexed in Science Citation Index (SCI) Expanded. Chin J Cancer. 2014;33:367-8. 